\title{
Edge Detection - Fuzzy Inference System
}

\author{
Er. Vishal Paika ${ }^{1,}$ Er. Pankaj Bhambri ${ }^{2}$ \\ ${ }^{1}$ Department of CSE, BCET Ludhiana \\ 1vishal.paika@gmail.com \\ ${ }^{2}$ Assitant Professor, Department of IT, GNDEC, Ludhiana
}

\begin{abstract}
In this paper a method has been developed for automatic edge detection of an digital image. An edge is made up of those pixels at which there is an abrupt change in the intensity. These pixels are known as edge pixels and are connected to give an edge. In this paper we have developed a mamdani's fuzzy inference system in MATLAB 2008 using fuzzy logic tool box. A smallest possible $2 X 2$ window is used as a scanning mask. Mask slides over the whole image pixel by pixel, first horizontally in topmost horizontal line then after reaching at the end of line, it increments to check the next vertical location and it continues till the whole image is scanned. The FIS built has 4 inputs, each input representing a pixel for $2 X 2$ mask, and 1 output that represents pixel under consideration. The rule editor consists of sixteen fuzzy rules. The results thus obtained are compared with Sobel edge operator and Canny edge operator.
\end{abstract}

Keywords: Digital image processing; Edge detection; Fuzzy Logic; Fuzzy inference system.

Academic discipline and sub-disciplines: Fuzzy Logic

Subject classification: Digital Image Processing

\section{Council for Innovative Research}

Peer Review Research Publishing System

Journal: International Journal of Management \& Information Technology

\author{
Vol.4, No.1 \\ editor@cirworld.com \\ www.cirworld.com, member.cirworld.com
}




\section{INTRODUCTION}

An image is a single picture which represents something. It may be a picture of a person, of people or animals, or of an outdoor scene, or a microphotograph of an electronic component, or the result of medical imaging. Digital image processing involves using a computer to change the nature of a digital image something like obtaining the edges of an image. Edge detection refers to the process of identifying and locating sharp discontinuities in an image. The discontinuities are abrupt changes in pixel intensity which characterize boundaries of objects in a scene. Discontinuities in image brightness are likely to correspond to discontinuities in depth, discontinuities in surface orientation, and changes in material properties or variations in scene illumination. The goal of edge detection is to locate the pixels in the image that correspond to the edges of the objects seen in the image. As edge detection is a fundamental step in computer vision, it is necessary to point out the true edges to get the best results from the matching process.

The edge detection has been used by object recognition, target tracking, segmentation, and etc. Therefore, the edge detection is one of the most important parts of image processing. It is an important concept both in the area of image processing and in the area of object recognition, Without being able to determine where the edge of an object fall, a machine would be able unable to determine many things about that object such as shape, volume, and area. Being able to recognize an object is a key step towards the development of artificial intelligence.

Shashank Mathur and Anil Ahlawat, presented an algorithm based on the subjection of a set of nine pixels, part of a $3 \times 3$ window of an image to a set of fuzzy conditions which help to highlight all the edges that are associated with an image. The fuzzy conditions test the relative values of pixels which can be present in case of presence of an edge. However their technique was not rule based [2]. Yinghua Li et.al. presented fuzzy technology as a newly rising technology used in many fields, especially in the image processing, and fuzzy enhancement as important part of fuzzy technology. In that paper firstly sets the image fuzzy feature plane of the original image, secondly proceeds the fuzzy enhancement, and then detects the edge by sobel differential arithmetic [3]. Yasar Becerikli1 and Tayfun of Kocaeli University, Computer Engineering Department, Izmit, Turkey proposed that an edge detection is one of the most important tasks in image processing as image segmentation, registration and identification are based on edge detection. They proposed that rulebased approach is advantages as it gives permission to adapt some parameters easily like the edges thickness can be changed easily by adding new rules or changing output parameters. They said that rule-based approach has flexible structure which can be adapted any time or any where easily and new fuzzy approach produces satisfactory result [4]. Human face is made up of eyes, nose, mouth and chin etc. There are differences in shape, size and structure of those organs, so the faces are differ in thousands ways, and we can describe them with the shape and structure of the organs so as to recognize them. One common method is to extract the shape of the eyes, nose, mouth and chin, and then distinguish the faces by distance and scale of those organs.

A very important role is played in image analysis by what are termed feature points, pixels that are identified as having a special property. Feature points include edge pixels as determined by the well-known classic edge detectors of Prewitt, Sobel, Marr, and Canny [7-11]. Recently there has been much revived interest in feature points determined by "corner" operators such as the Plessey, and interesting point operators such as that introduced by Moravec. Classical operators identify a pixel as a particular class of feature point by carrying out some series of operations within a window centred on the pixel under scrutiny. The classic operators work well in circumstances where the area of the image under study is of high contrast. In fact, classic operators work very well within regions of an image that can be simply converted into binary image by simple thresh holding.

Recent research has concerned Fuzzy Logic to develop edge detectors. The work of this paper is concerned with the development of a fuzzy logic rules based algorithm for the detection of edges in an image. A smallest possible scanning mask of size $2 * 2$ pixels window is used. Fuzzy Inference based system in MATLAB environment has been developed, which is capable of detecting edges of an image. The rule-base of sixteen rules has been designed to mark the pixel under consideration as Black, White or Edge. The noise removal algorithm has been implemented at intermediate and final levels of processing to get clear and sharp edges. The result has been compared with the standard algorithms.

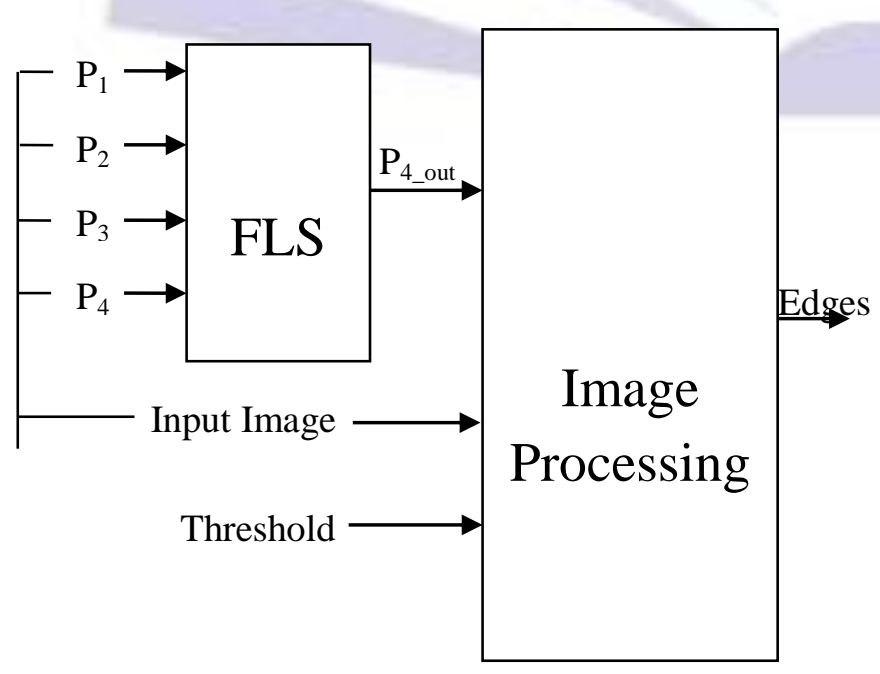

Fig. 1. Basic Block Diagram 


\section{FUZZY INFERENCE SYSTEM}

This fuzzy rule based edge detection system is developed by designing a Fuzzy Inference System (FIS) of Mamdani type using MATLAB 2008. A smallest possible 2X2 window is used as a scanning mask as shown in figure below: -

\begin{tabular}{|c|c|}
\hline$P_{1}$ & $P_{2}$ \\
$Y(i, j)$ & $Y(i, j+1)$ \\
\hline$P_{3}$ & $P_{4}$ \\
$Y(i+1, j)$ & $Y(i+1, j+1)$ \\
\hline 2×2 mask used for scanning \\
\hline
\end{tabular}

Mask slides over the whole image pixel by pixel, first horizontally in topmost horizontal line then after reaching at the end of line, it increments to check the next vertical location and it continues till the whole image is scanned. The FIS built has 4 inputs, each input representing a pixel for $2 X 2$ mask, and 1 output that represents pixel under consideration. The rule editor consists of sixteen fuzzy rules for considering the weights of like $P_{1}, P_{2}, P_{3}$ and $P_{4}$ with $P_{4}$ out for example if $P_{1}, P_{2}$, $\mathrm{P}_{3}$ and $\mathrm{P}_{4}$ are black then the $\mathrm{P}_{4 \_ \text {out }}$ is black. Figure 2 shows the rule editor.

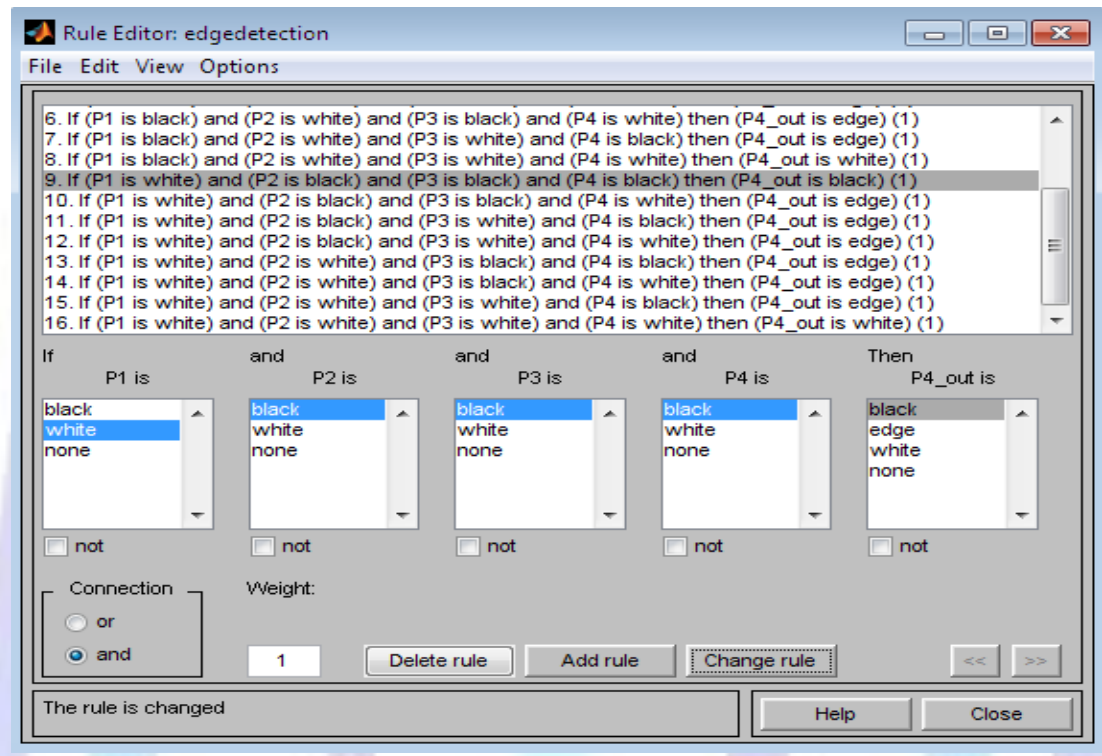

Fig. 2. Rule Editor

Fuzzy Logic Toolbox ${ }^{\mathrm{TM}}$ provides functions, apps, and a Simulink $\AA^{\circledR}$ block for analyzing, designing, and simulating systems based on fuzzy logic. The product guides you through the steps of designing fuzzy inference systems. Functions are provided for many common methods, including fuzzy clustering and adaptive neuro-fuzzy learning. The toolbox lets you model complex system behaviors using simple logic rules, and then implements these rules in a fuzzy inference system. See below mentioned figure 3 .
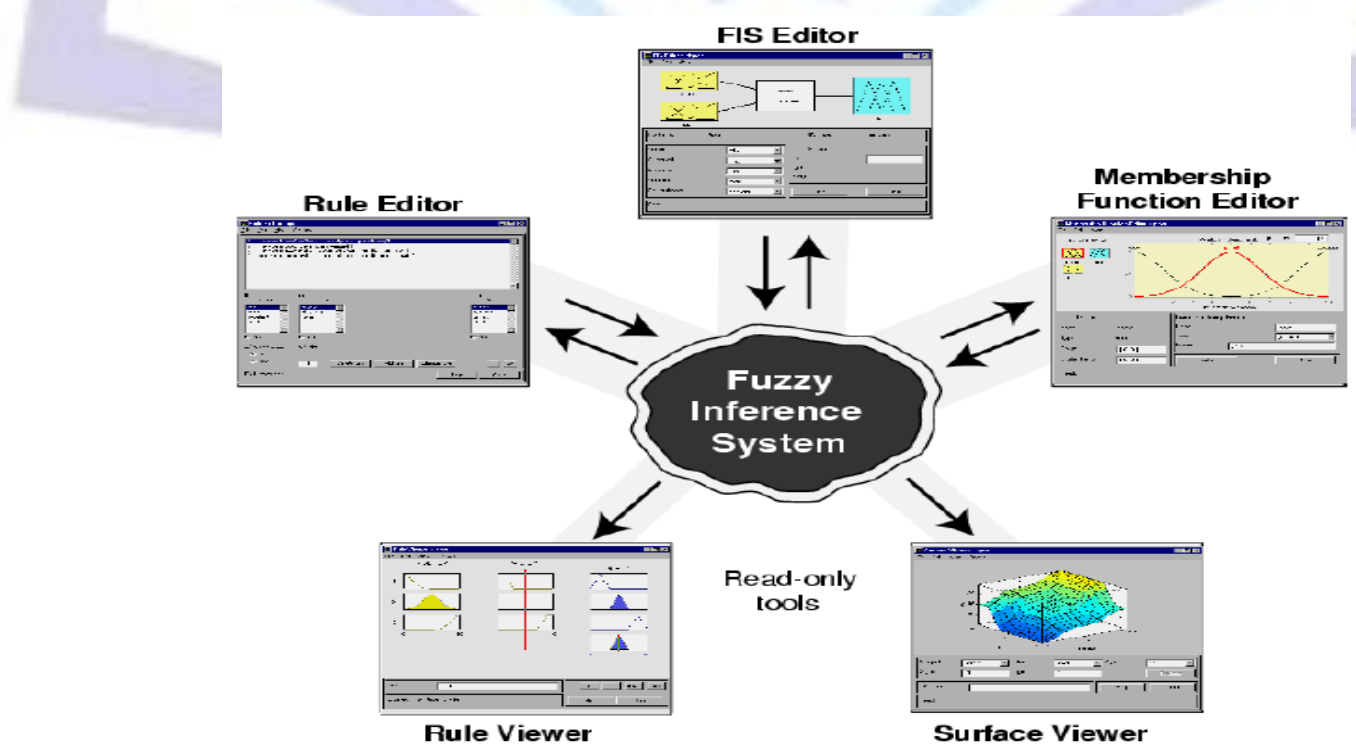

Fig. 3. Block diagram of FIS Editor 
Fuzzy logic is conceptually easy to understand and is flexible. The mathematical concepts behind fuzzy reasoning are very simple. Fuzzy logic is tolerant of imprecise data and Fuzzy logic can model nonlinear functions of arbitrary complexity. Fuzzy logic is based on natural language i.e. it is built atop the structures of qualitative description used in everyday language, fuzzy logic is easy to use. In general, the fuzzy logic provides an inference structure that enables appropriate human reasoning capabilities.

On the contrast, in classical set theory i.e. traditional binary set theory, the membership of elements in a set is assessed in binary terms according to a bivalent condition i.e. follows two valued logic, either true or false - an element either belongs or does not belong to the set. It describes crisp i.e. exact events, events that either do or do not occur. Consider ring an example that if ' $a$ ' is member or element of the set ' $A$ ' then only two possible relationships can exists between ' $a$ ' and ' $A$ ', either ' $a$ ' belongs to ' $A$ ' or ' $a$ ' does not belongs to ' $A$ '.

The theory of fuzzy logic is based upon the notion of relative graded membership and so are the functions of mutation and cognitive processes. The utility of fuzzy sets lies in their ability to model uncertain or ambiguous data, figure 4, so often encountered in real life. The Fuzzification and Defuzzification steps are due to the fact that we don't possess hardware.

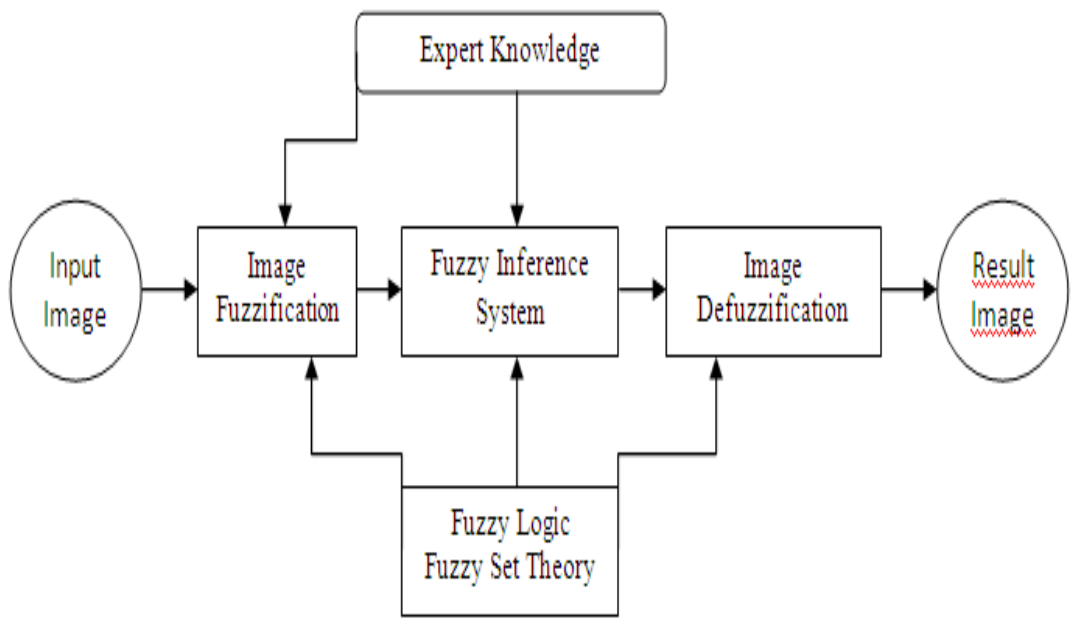

Fig. 4. Fuzzy Image processing system

In this paper, the designed fuzzy inference system is given four inputs i.e. $P_{1}, P_{2}, P_{3}, P_{4}$ and one output i.e. $P_{4}$ out. The four inputs are the four pixel values of the $2 X 2$ windows mask used. The trapezodial membership functions are used both for the inputs and the output. Two fuzzy sets i.e. Black and White are used for the input and three fuzzy sets i.e. Black, White and Edge are used for the output. Fuzzy sets for input and output variables are designed as shown in the figures below i.e. figure $5 . a$ and figure 5.b:

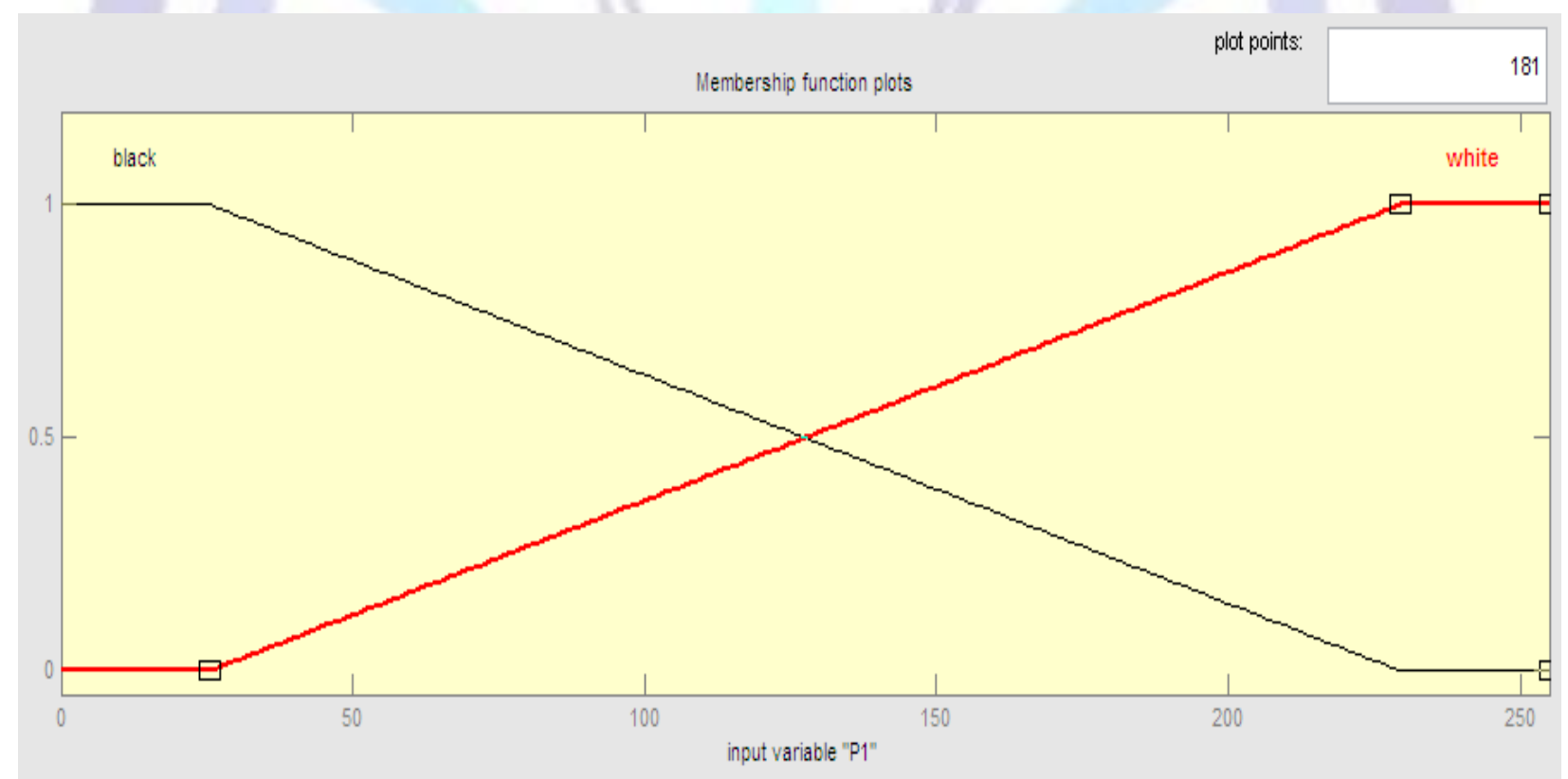

Fig. 5.a. Fuzzy input membership function for $P_{1}$ to $P_{4}$ 


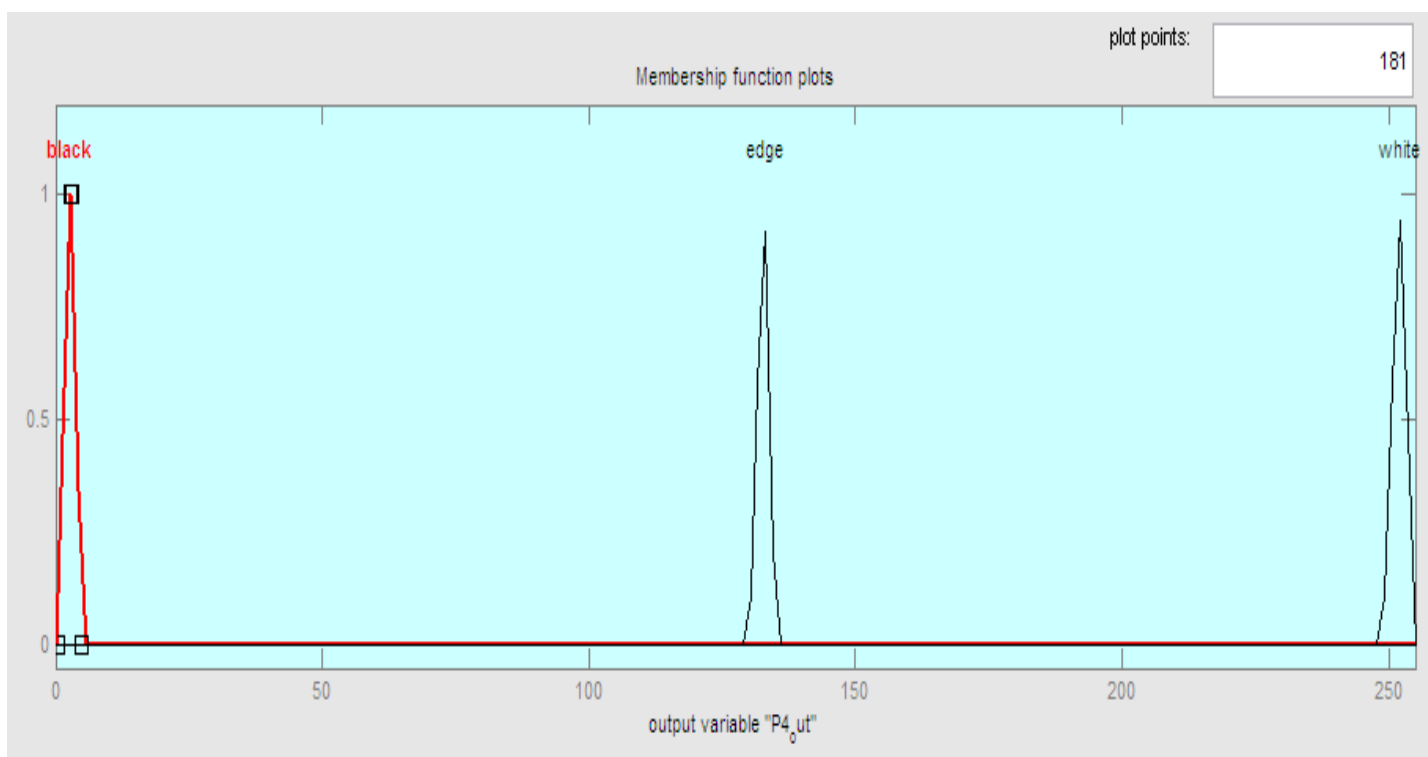

Fig. 5.b. Fuzzy output membership function for $\mathrm{P}_{4 \_ \text {_out }}$

Table I and Table II show the fuzzy sets for I/O variables and fuzzy rule matrix respectively. A rule base of 16 rules is set for the various fuzzy conditions that can occur. Singe output describes whether the output pixel i.e. $P_{4 \_ \text {out }}$ belongs to White fuzzy set, Black fuzzy set or Edge fuzzy set. Rules are enlisted in the form of a matrix in Table. II

\begin{tabular}{|c|c|c|}
\hline \multicolumn{3}{|c|}{ Fuzzy input no. 1, Pixel P1 } \\
\hline Name & Range & MF Type \\
\hline Black & {$\left[\begin{array}{lllll}0 & 0 & 25.5 & 22 & 29.5\end{array}\right]$} & Trapezodial \\
\hline White & {$\left[\begin{array}{lllll}25.5 & 229.5 & 255 & 255\end{array}\right]$} & Trapezodial \\
\hline \multicolumn{3}{|c|}{ Fuzzy input no. 2, Pixel P2 } \\
\hline Black & {$\left[\begin{array}{lllll}0 & 0 & 25.5 & 229.5\end{array}\right]$} & Trapezodial \\
\hline White & {$\left[\begin{array}{lllll}25 & 5 & 229.5 & 255 & 255\end{array}\right]$} & Trapezodial \\
\hline \multicolumn{3}{|c|}{ Fuzzy input no. 3, Pixel P3 } \\
\hline Black & {$\left[\begin{array}{llllll}0 & 0 & 25 & 5 & 2 & 229.5\end{array}\right]$} & Trapezodial \\
\hline White & {$\left[\begin{array}{lllll}25.5 & 229.5 & 255 & 255\end{array}\right]$} & Trapezodial \\
\hline \multicolumn{3}{|c|}{ Fuzzy input no. 4, Pixel P4 } \\
\hline Black & {$\left[\begin{array}{llllll}0 & 0 & 25.5 & 22 & 29.5\end{array}\right]$} & Trapezodial \\
\hline White & {$\left[\begin{array}{lllll}25.5 & 229.5 & 255 & 255\end{array}\right]$} & Trapezodial \\
\hline \multicolumn{3}{|c|}{ Fuzzy output no. 1, Pixel P4_out } \\
\hline Black & {$[0.32 .73 .35 .7]$} & Trapezodial \\
\hline Edge & {$\left[\begin{array}{lllll}130.3 & 132.7 & 133.2 & 134.8\end{array}\right]$} & Trapezodial \\
\hline White & {$[249.3251 .7252 .3254 .7]$} & Trapezodial \\
\hline
\end{tabular}




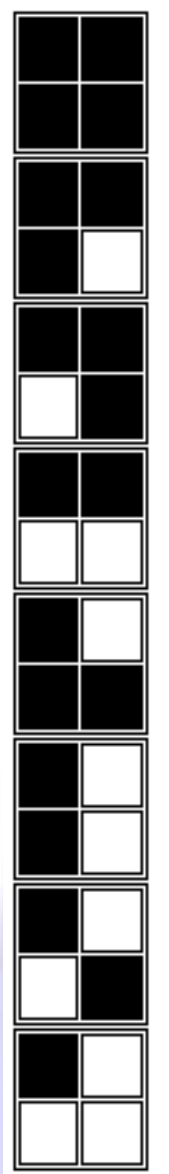

Rule 1: If $\{Y(i, j) \& Y(i, j+1)\}$ are Blacks If $\{Y(i+1, j) Y(i+1, j+1)\}$ are Blacks

Then checked pixel $\mathrm{P}_{4}$ is a Black.

Rule 2: If $\{Y(i, j) \& Y(i, j+1) \& Y(i+1, j)\}$ are Blacks If $\{Y(i+1, j+1)\}$ is White

Then checked pixel $\mathrm{P}_{4}$ is an Edge.

Rule 3: If $\{Y(i, j) \& Y(i, j+1) \& Y(i+1, j+1)\}$ are Blacks If $\{Y(i+1, j)\}$ is White

Then checked pixel $\mathrm{P}_{4}$ is an Edge.

Rule 4: If $\{Y(i, j) \& Y(i, j+1)\}$ are Blacks If $\{Y(i+1, j) \& Y(i+1, j+1)\}$ are Whites

Then checked pixel $\mathrm{P}_{4}$ is an Edge.

Rule 5 : If $\{Y(i, j) \& Y(i+1, j) \& Y(i+1, j+1)\}$ are Blacks If $\{Y(i, j+1)\}$ is White

Then checked pixel $\mathrm{P}_{4}$ is an Edge.

Rule 6: If $\{Y(i, j) \& Y(i+1, j)\}$ are Blacks If $\{Y(i, j+1) \& Y(i+1, j+1)\}$ are Whites Then checked pixel $\mathrm{P}_{4}$ is an Edge.

Rule 7: If $\{Y(i, j) \& Y(i+1, j+1)\}$ are Blacks If $\{Y(i, j+1) \& Y(i+1, j)\}$ are Whites Then checked pixel $\mathrm{P}_{4}$ is an Edge.

Rule 8: If $\{Y(i, j)\}$ is Black If $\{Y(i, j+1) \& Y(i+1, j) \& Y(i+1, j+1)\}$ are Whites Then checked pixel $\mathrm{P}_{4}$ is a White.

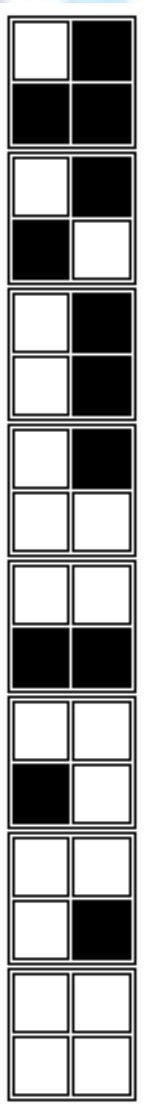

Rule 9: If $\{Y(i, j+1) \& Y(i+1, j) \& Y(i+1, j+1)\}$ are Blacks If $\{Y(i, j)\}$ is White

Then checked pixel $\mathrm{P}_{4}$ is a Black

Rule 10: If $\{Y(i, j+1) \& Y(i+1, j)\}$ are Blacks If $\{Y(i, j) \& Y(i+1, j+1)\}$ are Whites Then checked pixe $1 \mathrm{P}_{4}$ is an Edge.

Rule 11: If $\{Y(i, j+1) \& Y(i+1, j+1)\}$ are Blacks If $\{Y(i, j) \& Y(i+1, j)\}$ are Whites Then checked pixe1 $\mathrm{P}_{4}$ is an Edge.

Rule 12 : If $\{Y(i, j+1)\}$ is Black If $\{Y(i, j) \& Y(i+1, j) \& Y(i+1, j+1)\}$ are Whites Then checked pixe1 $\mathrm{P}_{4}$ is an Edge.

Rule 13: If $\{Y(i+1, j) \& Y(i+1, j+1)\}$ are Blacks If $\{Y(i, j) \& Y(i, j+1)\}$ are Whites Then checked pixe1 $\mathrm{P}_{4}$ is an Edge.

Rule 14: If $\{Y(i+1, j)\}$ is Black If $\{Y(i, j) \& Y(i, j+1) \& Y(i+1, j+1)\}$ are Whites Then checked pixe1 $\mathrm{P}_{4}$ is an Edge.

Rule 15 : If $\{Y(i+1, j+1)\}$ is Black If $\{Y(i, j) \& Y(i, j+1) \& Y(i+1, j)\}$ are Whites Then checked pixe $1 \mathrm{P}_{4}$ is an Edge.

Rule 16: If $\{Y(i, j) \& Y(i, j+1)\}$ are whites If $\{Y(i+1, j) \& Y(i+1, j+1)\}$ are Whites Then checked pixe $1 \mathrm{P}_{4}$ is a White.

TABLE II FUZZY RULE BASE 


\section{EXPERIMENTS}

The implementation was carried out consisdering that the input image and the output image are both 8-bit quantized so that their gray levels are always between 0 and 255. The condition of each pixel is decided by using a floating mask window of 2X2 size which will scanning all the grays. In rule number 1 and 9 , the checked pixel is black, in rule number 8 and 16 the checked pixel is white and in rule numbers from 2 to 7 and 10 to 15, the checked pixel corresponds to edge. In fuzzification the input grays are ranged from 0 to 255 gray intensity and in the defuzziaction again the vlaues range from 0 to 255 gray intensity levels. From the tested images in this we found that best results are achived at the range black from 0 to 25 gray valus and from 230 to 255 meaning that the weight is white.

The fuzzy conditions help us to test the relative values of pixels which can be present in case of presence on an edge. So the relative pixel values are instrumental in extracting all the edges associated to an image. The image is said to have an edge if the intensity variation in between the adjacent pixels is large. Edge Detection - Locating areas with strong intensity contrasts. The mask is slid over an area of the input image, changes that $\mathrm{P}_{4}$ pixel's value and then shifts one pixel to the right and continues to the right until it reaches the end of a row. It then starts at the beginning of the next row and process continues till the whole image is scanned.

When this mask is made to slid over the image, the output is generated by the fuzzy inference system based upon the sixteen rules and the value of the pixels $P_{1}, P_{2}, P_{3}$ and $P_{4}$ say for example rule number 4 states that when the values of pixels $P_{1}$ and $P_{2}$ are black and that of pixels $P_{3}$ and $P_{4}$ are white then the output $P_{4}$ out is an edge. The FIS system was tested with different type of face images and its performance was compared with the standard sobel edge detection algorithm and canny edge detection algorithm.

\section{RESULTS}

The proposed system was tested with different images, its performance being compared to that of the Sobel edge detection algorithm and Canny edge detection algorithm in MATLAB environment. The firing order associated with each fuzzy rule were tuned to obtain good results while extracting edges of the image where we used this image as comparative model for the classical Sobel operator, Canny operator and the FIS method. Results of different images are shown below. Figure 6.a, 7.a and 8.a shows the original image in gray scale form, which is obtained after conversion from the colour image captured from any image capturing device, figure 6.b, 7.b and 8.b shows the edges using the classical Sobel operator, figure 6.c, 7.c and 8.c shows the edges using the Canny edge detection operator and figure 6.d, 7.d and 8.d shows the edges of our designed Fuzzy Inference System.

From figure 6.b, 7.b and 8.b, 6.c, 7.c and 8.c \& 6.d, 7.d and 8.d, it is observed that the output that has been generated by the fuzzy method has found out the edges of the image more distinctly as compared to the ones that have been found out by the "Sobel" edge detection algorithm and "Canny" edge detection algorithm. Observe that our FIS has caputured not only sharp transition from high intensity level to low intensity level (i.e from black to gray) but is has captured even a small change in the intensity variation. Thus the Fuzzy rule based algorithm provides better edge detection and has an exhaustive set of fuzzy conditions which helps to extract the edges with a very high efficiency.

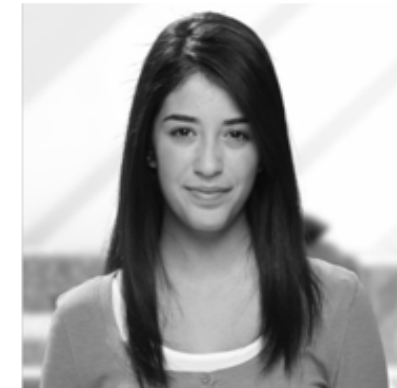

Figure 6 (a)

Original Gray Scale Image

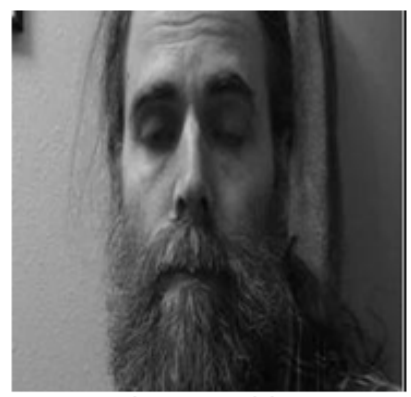

Figure 7 (a)

Original Gray Scale Image

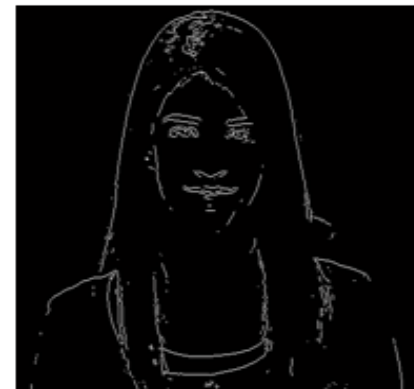

Figure 6(b)

Edges using Sobel Operator

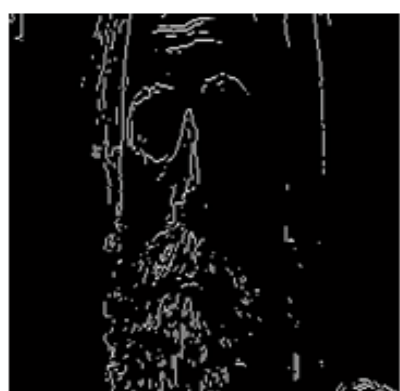

$\begin{array}{cc}\text { Figure } 7 \text { (b) } & \text { Figure } 7 \text { (c) } \\ \text { Edges using Sobel Operator } & \text { Edges using Canny Operator }\end{array}$

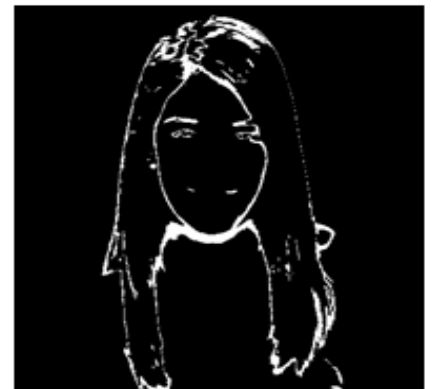

Figure 6 (d)

Figure 6 (c)

Edges using Canny Operator

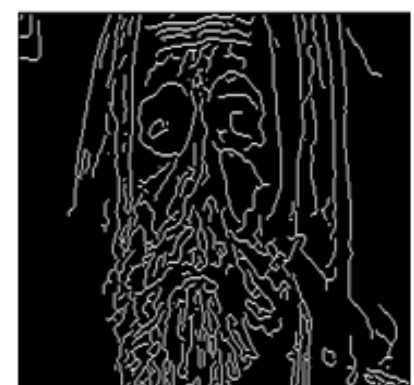

Figure 7 (c)

Edges using FIS

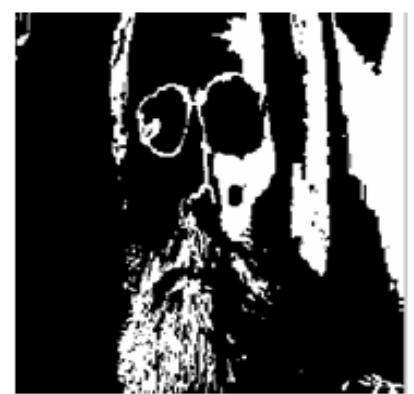

Figure 7 (d)

Edges using FIS 


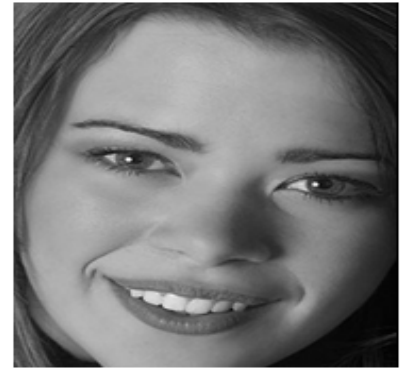

Figure 8 (a)

Original Gray Scale Image

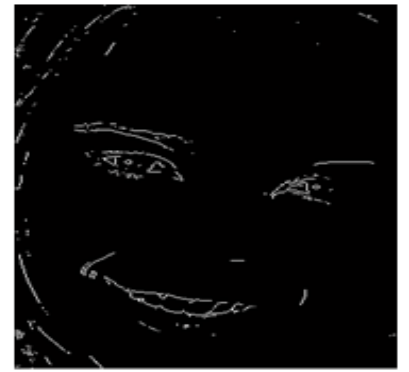

Figure 8 (b)

Edges using Sobel Operator Edges using Canny Operator

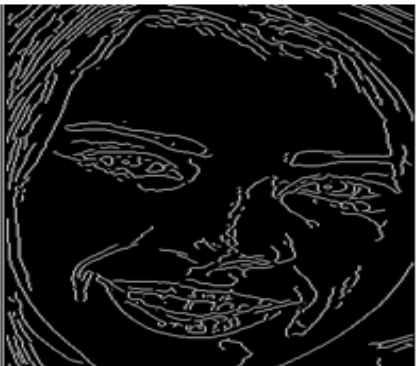

Figure 8 (c)

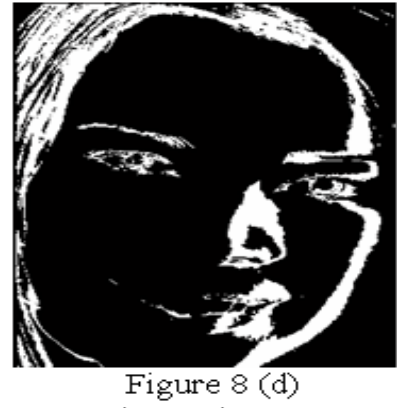

Edges using FIS

\section{CONCLUSION}

Because of the uncertainties that exist in many aspects of image processing, fuzzy processing is desirable. These uncertainties include additive and non-additive noise in lowlevel image processing, imprecision in the assumptions underlying the algorithms, and ambiguities in interpretation during high level image processing.

In this paper, emphasis has been to develop a very simple and small but a very efficient, fuzzy rule based edge detection algorithm to abridge the concepts of artificial intelligence and digital image processing. We have used the smallest possible mask of size $2 \times 2$. The algorithm has been developed in MATLAB environment. Comparisons were made with the various other edge detection algorithms that have already been developed like sobel edge detection algorithm and canny edge detection algorithm. Displayed results have shown the accuracy of the edge detection using the fuzzy rule based algorithm over the other algorithms.

The fuzzy rule based algorithm has been successful in obtaining the edges that are present in an image after its implementation and execution with various sets of images. Threee sample outputs, all human faces, have been shown to make the readers understand the accuracy of the algorithm. Thus developed algorithm exhibits tremendous scope of application in various areas of digital image processing.

\section{FUTURE WORK}

a) In our technique, the image is first to be converted into gray image. This limitation can be eliminated and algorithm can be applied directly to color images, and the detection would then become significantly more complex.

b) Further the size of mask can be increased from $2 \times 2$ to $3 \times 3$ or $4 \times 4$ and accordingly more rules can be set and results can be compared with respect to the mask size.

c) In our method, we have used Mamdani Fuzzy Inference System. But we can also use Sugeno FIS and compare both the results.

\section{REFERENCES}

[1] Y. Kuo, C. Lee, and C. Liu, "A New Fuzzy Edge Detection Method for image Enhancement”, IEEE Inter. Conf. on Fuzzy Systems, 1997, pp. 1069-1074.

[2] Shashank Mathur, Anil Ahlawat, "Application Of Fuzzy Logic In Image Detection", International Conference "Intelligent Information and Engineering Systems" INFOS 2008, Varna, Bulgaria, June-July 2008

[3] Yinghua Li, Bingqi Liu, and Bin Zhou, "The Application Of Image Edge Detection by using Fuzzy Technique", in Conference "Electronic Imaging and Multimedia Technology", November 2004.

[4] Yasar Becerikli1 and Tayfun M. Karan, "A New Fuzzy Approach for Edge Detection", Computational Intelligence and Bio inspired Systems", June 2005.

[5] D.O Aborisade, "Novel Fuzzy logic Based Edge Detection Technique", International Journal of Advanced Science and Technology Vol. 29, April 2011.

[6] Abdallah A. Alshennawy, and Ayman A. Aly, "Edge Detection in Digital Images Using Fuzzy Logic Technique", World Academy of Science, Engineering and Technology 512009.

[7] G.I. Sanchez-Ortiz, A. Noble,"Fuzzy clustering driven anisotropic diffusion: enhancement and segmentation of cardiac MR images", Nuclear Science Symposium, Vol. 3, 1998, pp. 1873 -1874.

[8] R.L. Webber, U.E. Ruttimann, and R.A.J. Groenhuis, "Computer correction of projective distortions in Dental Radiographs", J. Den. Res., vol. 63, No. 8, pp.1032-1036, August 1984.

[9] Song Wang, Feng Ge, Tiecheng Liu, "Evaluating Edge Detection Through Boundary Detection", Department of Computer Science and Engineering, University of South Carolina, Columbia, SC 29208, USA, 2006.

[10] Ivan Christov, " Multiscale Image Edge Detection", 1.130/18.327, Spring 2004, Final Project, May 12, 2004.

[11] Nor Ashidi Mat Isa, " Automated Edge Detection Technique for Pap Smear Images Using Moving K-Means Clustering and Modified Seed Based Region Growing Algorithm", International Journal of The Computer, the Internet and Management Vol. 13.No.3 (September-December, 2005) pp 45-59.

[12] S. N. Sivanandam, S. Sumathi and S. N. Deepa, "Introduction to Fuzzy Logic using MATLAB". 